\title{
Adjustment of Overestimated CT-Based Attenuation Correction on Bone SPECT/CT After Hip-Resurfacing Arthroplasty
}

\author{
Akira Suzuki $^{1,2}$, Kichiro Koshida ${ }^{3}$, and Kosuke Matsubara ${ }^{3}$ \\ ${ }^{I}$ Diagnostic Imaging Center, Japanese Red Cross Sendai Hospital, Miyagi, Japan; ${ }^{2}$ Division of Health Sciences, Graduate School of \\ Medical Sciences, Kanazawa University, Kanazawa, Japan; and ${ }^{3}$ Department of Quantum Medical Technology, Faculty of Health \\ Sciences, Institute of Medical, Pharmaceutical, and Health Sciences, Kanazawa University, Kanazawa, Japan
}

SPECT/CT has made it possible to perform attenuation correction easily. However, CT-based attenuation correction (CTAC) is overestimated when metal is inside the body. The aim of this investigation was to create and test a new attenuation correction formula for CTAC that decreases the overestimation caused by an artificial femoral head and hip joint. Methods: We tested the usefulness of the new formula in a phantom study. The phantom contained "bones" made from gypsum. The components were placed in the phantom, the point source was placed both near metal and away from metal, and the counts were compared to verify the usefulness of the new attenuation correction formula. The new formula included use of thresholds to prevent the overestimation caused by proximity to metal. Results: With the standard formula, the maximum overestimation was $18 \%$ for a $22-\mathrm{cm}$ artificial hip joint placed in the acetabular roof. With the new formula, using 2 thresholds, the overestimation decreased to $4 \%$. Conclusion: The new attenuation correction formula helps provide more correct data in SPECT/CT examinations of patients with metallic implants.

Key Words: SPECT/CT; CTAC; bone; metal

J Nucl Med Technol 2013; 41:203-207

DOI: $10.2967 /$ jnmt.113.121152

\section{W}

hole-body scintigraphy is frequently used to examine bones because it can easily assess the integrity of the entire skeleton (1). The number of scintigraphic bone examinations has risen with the increase in the incidence of breast and prostate cancer. The development of SPECT/ $\mathrm{CT}$ led to the expectation that the CT data, besides providing anatomic information, would provide the data from which an attenuation map can be created for correction of

\footnotetext{
Received Feb. 7, 2013; revision accepted Jun. 24, 2013.

For correspondence or reprints contact: Akira Suzuki, Diagnostic Imaging Center, Japanese Red Cross Sendai Hospital, 43-3 Yagiyamahonntyou2chome, Sendashi, Miyagi 982-8501, Japan.

E-mail: hokumeiryou@hotmail.co.jp

Published online Aug. 5, 2013.

COPYRIGHT @ 2013 by the Society of Nuclear Medicine and Molecular Imaging, Inc.
}

the SPECT data (2-7). Currently, a bilinear function is used in attenuation correction formulas for SPECT/CT (2). When the CT value is less than 0 , the attenuation coefficient of a nuclide is calculated using a mixed model of water and air, and when the CT value is 0 or more, the calculation is done using a mixed model of water and bone. The main factors in attenuation are a photoelectric effect and Compton scattering: with low energy, the photoelectric effect is the main factor in attenuation, and with high energy, Compton scattering is the main factor. For this reason, when the lower energy of CT is used for attenuation correction, the presence of high-atomic-number metals results in attenuation estimates that are more than the actual SPECT attenuation. In fact, when there is metal inside the body, CT-based attenuation correction (CTAC) has been found to be inaccurate (8). Hip-resurfacing arthroplasty is a common procedure, and it is likely that patients who receive an artificial femoral head will undergo bone scintigraphy. Presently, even if a SPECT/CT examination is needed to evaluate bone cancer, it cannot be performed correctly using CTAC when an artificial femoral head is present. To our knowledge, no one has yet reported a formula by which the overestimation can be corrected. The aim of this investigation was to develop and test a formula for CTAC that decreases the overestimation caused by the presence of an artificial hip joint and femoral head.

\section{MATERIALS AND METHODS}

Imaging

A phantom was made from a plastic container, water, and artificial bones, along with a plinth of wax supporting them. The bones consisted of gypsum, because it can be distinguished from contrast medium on imaging. The stems of the bones were of 2 types: VerSys Fiber Metal MidCoat (HA/TCP) and VerSys Fiber Metal Taper (HA/TCP), both manufactured by Zimmer. Both types were $30.5 \mathrm{~cm}$ (12 in) long. The femoral head (Durasul Biopolar; Zimmer) had a cup size of $36 \mathrm{~cm}$. The hip joint (Trilogy Acetabular Cup; Zimmer) had a cup size of $22 \mathrm{~cm}$. The point source was rectangular $(3 \times 1 \mathrm{~cm})$ filter paper permeated with ${ }^{99 \mathrm{~m}} \mathrm{Tc}$-pertechnetate. The configuration of the phantom and point source is shown in Figure 1. For 


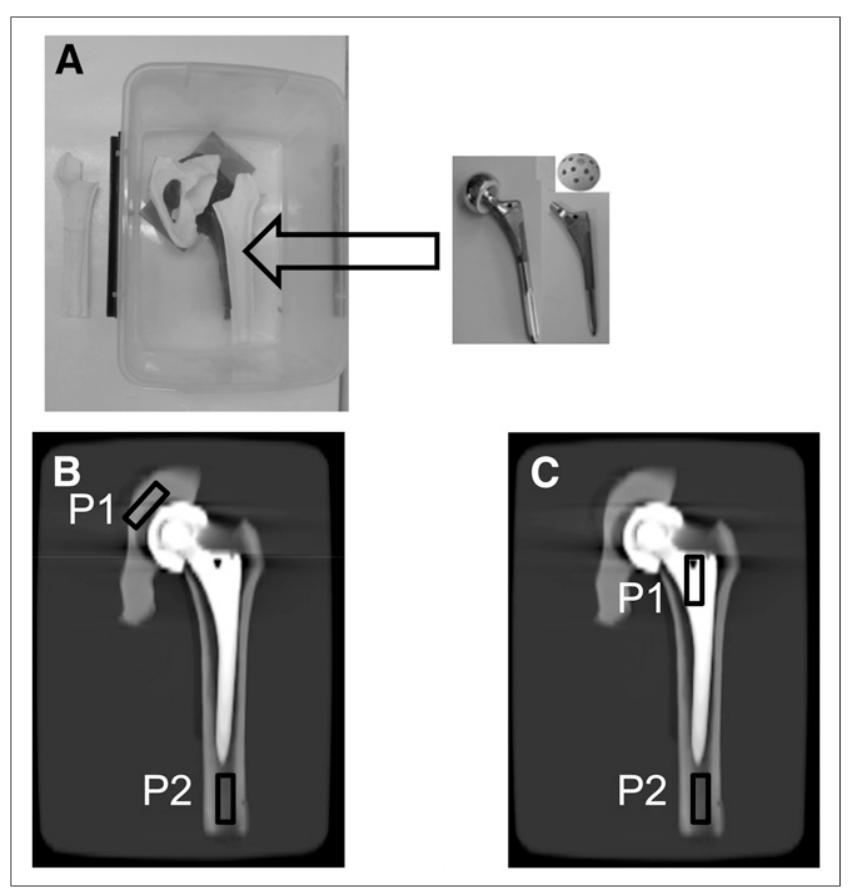

FIGURE 1. Configuration of phantom and point source: photograph of phantom (A), location of point source for investigating influence of artificial hip joint and femoral head (B), and location of point source for investigating influence of stems (C). P1 = point source 1; P2 = point source 2 .

each experiment, a single point source 1 was placed in the acetabular roof and femur at the point of overlap with metal. Point source 2 was placed at the same location in each experiment-one that did not overlap with metal. Both point source 1 and point source 2 contained the same dose of tracer (mean administered activity, $4.5 \pm 1.3 \mathrm{MBq}$ ). SPECT/CT imaging was performed on a Symbia T2 scanner (Siemens Medical Solutions), which consists of a pair of low-energy, high-resolution collimators and a dual-head $\gamma$-camera and CT scanner. The matrix was $128 \times 128$, the angular step was 32 , and the time per frame was $20 \mathrm{~s}$. Counts from the $15 \%$ energy window at $140 \mathrm{keV}$ were acquired at a magnification of 1 . CT scanning was performed from the head to the pelvic floor using $140 \mathrm{kV}$,
$80 \mathrm{~mA}$, an 0.8 -s tube rotation time, a pitch of 1.2 , and a $5-\mathrm{mm}$ $(2 \times 2.5)$ slice thickness. Data were processed by interactive reconstruction on a Syngo workstation (Siemens Medical Solutions). The proposed attenuation correction formula was also created on the same workstation. Images were reconstructed using 3-dimensional ordered-subsets expectation maximization.

\section{Creation of Formula}

In CTAC, the ideal would be for suitable attenuation coefficients to be assigned to every organ (segmentation). However, segmentation from CT values is complex and is difficult for an analysis system to achieve. Because of beam hardening, CT values differ in the same organs. And although a CT value of 0 has been defined for water, there is no definition for the CT value of other substances. Therefore, the CT values for the same bone will be different for each type of CT scanner. Segmentation is also difficult because of the partial-volume effect. For this reason, current systems use a bilinear method to create the formula for CTAC. The formula takes into account 2 regions: the air-to-water region $(\mathrm{CT}$ value $\leq 0)$ and the water-to-bone (metal, etc.) region $(\mathrm{CT}$ value $>0)$.

Because the effective energies of CT and SPECT differ, the attenuation coefficient differs for substances of high atomic number, and we believe that overestimation occurs in the presence of metal because the attenuation coefficient becomes higher than needed. We assigned a threshold value to the attenuation coefficient formula to control for overestimation due to metal. The CT value is higher for bone and metal than for other internal organs. Therefore, assigning a threshold to a high CT value does not affect the attenuation coefficient of other internal organs. Although this attenuation coefficient is unchangeable, a user can alter the attenuation correction formula that changes CT values into an attenuation coefficient. Changing an attenuation coefficient map ( $\mu$-map) can demonstrate the same effect as does the attenuation correction formula.

We created 2 kinds of $\mu$-map: one with a single threshold and another with a double threshold. $\mu$-maps with a single threshold were set up assuming the presence of metal. $\mu$-maps with a double threshold assumed the presence of both bone (low threshold) and metal (high threshold). Because we wanted to clearly differentiate the attenuation coefficients of bone and metal, we used the threshold

FIGURE 2. Graph of attenuation correction formula with 1 threshold.

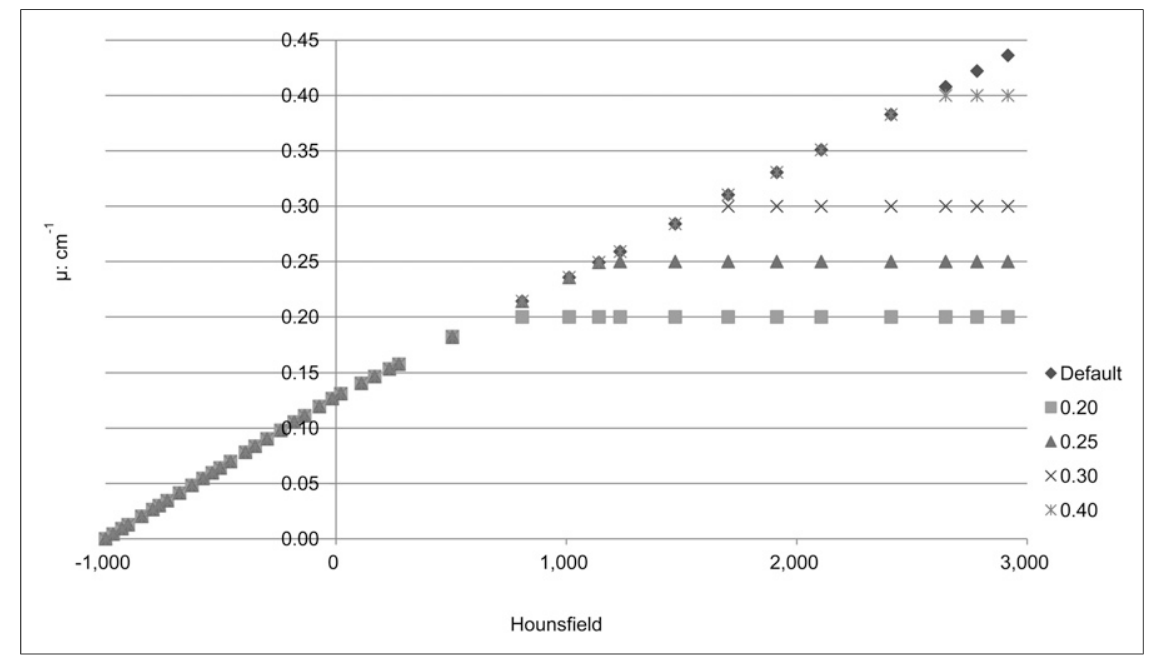




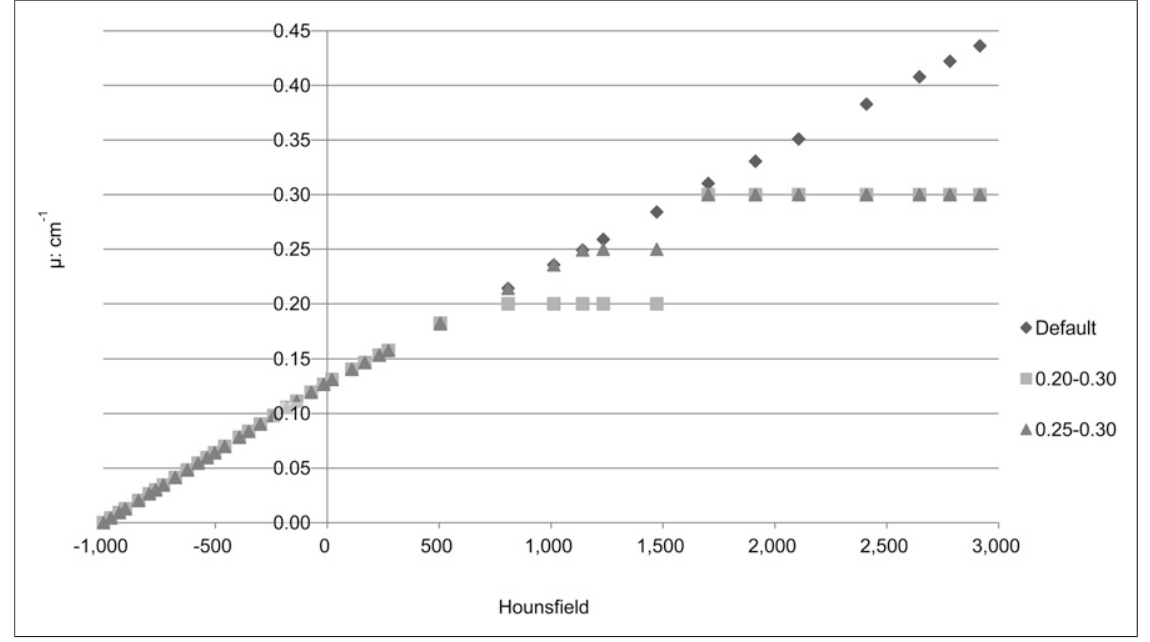

FIGURE 3. Graph of attenuation correction formula with 2 thresholds. for bone. This method is the same as the segmentation method for distinguishing bone and metal from other organs. If 2 thresholds are set, the attenuation correction formula turns into a hybrid formula incorporating both the bilinear method and the segmentation method. The formula is as follows:

$$
\begin{aligned}
& \text { Result } 1=(1-((0.0001-(\mathrm{A}-\mathrm{B})) \times 1,000)) \times \mathrm{B}, \\
& \text { Result } 2=\mathrm{A}-(\mathrm{A}-\mathrm{C}) \\
& \text { Result } 3=\text { result } 2 \times(0.0001-(\mathrm{A}-\mathrm{B})), \\
& \text { Result } 4=\text { result } 1+\text { result } 3,
\end{aligned}
$$

where $\mathrm{A}$ is the value of the original $\mu$-map, $\mathrm{B}$ is the threshold value supposing metal, $\mathrm{C}$ is the threshold value supposing bone ( $\mathrm{B}$ is substituted for $\mathrm{C}$ when there is 1 threshold), and result 4 is the value of the new $\mu$-map.

In the original $\mu$-map, all values greater than $B$ are set to $B$ and all values greater than $C$ but less than $B$ are set to $C$. Single thresholds were set at attenuation coefficients of $0.20,0.25,0.30$, and 0.40 . Double thresholds were set at 0.20 and 0.30 and at 0.25 and 0.30 .

\section{Testing of Formula}

When metal was present, the ratio of the count of point source 1 to the count of point source 2 was measured. The counts of the point sources were analyzed using paired $t$ tests.

We compared the ratio of the count of SPECT with no attenuation correction, SPECT corrected using the standard (default) CTAC formula, and SPECT corrected using the new CTAC formula. Thereby, the effect of the new formula was investigated.

\section{RESULTS}

Figure 2 illustrates use of single thresholds, and Figure 3 , double thresholds. When metal was present, the ratio of the count of point source 1 to the count of point source 2 was calculated. The results are shown in Table 1. The result without attenuation correction was poor. Use of the default formula resulted in overestimation, although this was good in comparison with the result when no attenuation correction was performed. When an artificial hip joint was set in an acetabular roof, the overestimation maximized at 20\%. A good result was obtained when

\begin{tabular}{|c|c|c|c|c|c|c|c|c|}
\hline \multirow[b]{2}{*}{ Site } & \multirow[b]{2}{*}{ Without AC } & \multirow[b]{2}{*}{ Default formula } & \multicolumn{6}{|c|}{ Formula using threshold of. .. } \\
\hline & & & 0.4 & 0.3 & 0.25 & 0.2 & 0.25 and 0.30 & 0.20 and 0.30 \\
\hline $\begin{array}{l}\text { Artificial femoral } \\
\text { head }\end{array}$ & $0.72 \pm 0.01^{*}$ & $1.18 \pm 0.03^{\star}$ & $1.14 \pm 0.03^{\dagger}$ & $1.07 \pm 0.03^{\dagger}$ & $1.02 \pm 0.03^{\ddagger}$ & $0.93 \pm 0.02^{\dagger}$ & $1.06 \pm 0.02^{\ddagger}$ & $1.02 \pm 0.02^{\ddagger}$ \\
\hline $\begin{array}{l}\text { Artificial hip } \\
\text { joint }\end{array}$ & $0.68 \pm 0.01^{*}$ & $1.20 \pm 0.02^{*}$ & $1.18 \pm 0.02^{*}$ & $1.12 \pm 0.02^{*}$ & $1.06 \pm 0.02^{\dagger}$ & $0.96 \pm 0.02^{\ddagger}$ & $1.11 \pm 0.02^{\star}$ & $1.04 \pm 0.02^{\ddagger}$ \\
\hline $\begin{array}{l}\text { VerSys Fiber } \\
\text { Metal MidCoat }\end{array}$ & $0.87 \pm 0.02^{*}$ & $1.11 \pm 0.02^{\dagger}$ & $1.08 \pm 0.02^{\dagger}$ & $1.02 \pm 0.01^{\ddagger}$ & $0.98 \pm 0.02^{\ddagger}$ & $0.92 \pm 0.02^{\dagger}$ & $1.01 \pm 0.02^{\ddagger}$ & $1.00 \pm 0.02^{\ddagger}$ \\
\hline $\begin{array}{l}\text { VerSys Fiber } \\
\text { Metal Taper }\end{array}$ & $0.85 \pm 0.02^{*}$ & $1.11 \pm 0.02^{\dagger}$ & $1.08 \pm 0.02^{\dagger}$ & $1.01 \pm 0.02^{\ddagger}$ & $0.97 \pm 0.02^{\ddagger}$ & $0.92 \pm 0.02^{\dagger}$ & $1.01 \pm 0.02^{\ddagger}$ & $0.98 \pm 0.02^{\ddagger}$ \\
\hline $\begin{array}{l}{ }^{\star} P<0.01 . \\
{ }^{\dagger} P<0.05 . \\
{ }^{\ddagger} \text { Not statistically } \\
\text { AC }=\text { attenuation }\end{array}$ & $\begin{array}{l}\text { ignificant ( } P \\
\text { correction. }\end{array}$ & $\geq 0.05$ ). & & & & & & \\
\hline
\end{tabular}
thresholds were set at 0.20 and 0.30 . Figure 4 shows an

TABLE 1

Ratio of Point Source 2 to Point Source 3 for Artificial Femoral Head and Hip Joint in Phantom 


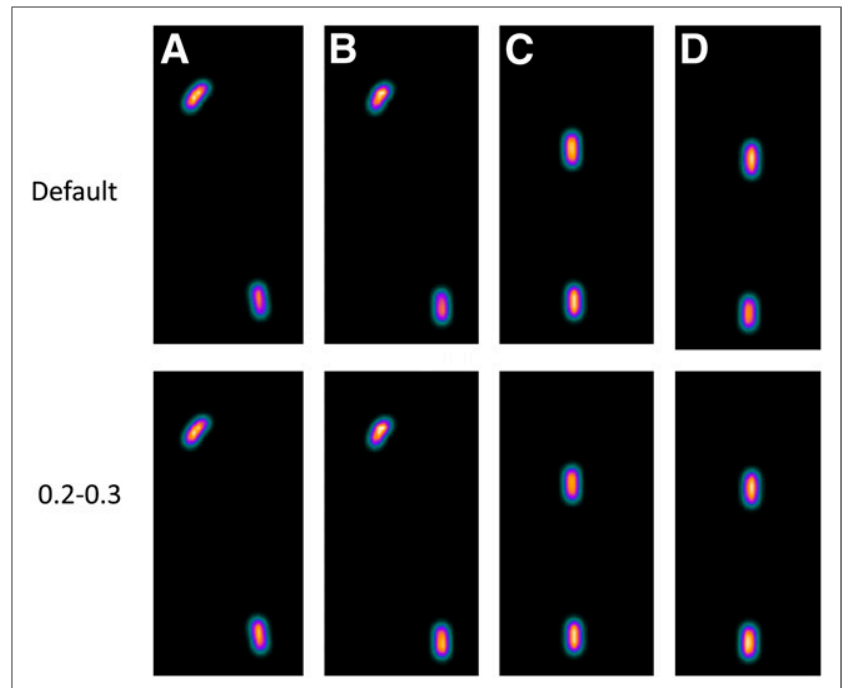

FIGURE 4. Comparison between image with no threshold and image with 2 thresholds: artificial femoral head (A), artificial hip joint (B), VerSys Fiber Metal MidCoat (C), and VerSys Fiber Metal Taper (D).

image with no threshold set and an image with thresholds of 0.20 and 0.30 . The paired $t$ test did not show a significant difference $(5 \%)$. Overestimation was controlled to a maximum of $4 \%$.

\section{DISCUSSION}

The anatomic information provided by SPECT is poor. Therefore, it is difficult to judge where the accumulation of tracer is. Besides this difficulty, the influence of attenuation on image findings is problematic, particularly in such modalities as myocardial perfusion SPECT. The development of SPECT/CT made it possible to combine the SPECT images with attenuation-corrected CT images and create a fusion of the two. Fusion images clearly show the location of any accumulation.

Accuracy is desirable in CTAC. The main factors in attenuation are a photoelectric effect and Compton scattering: with low energy, the photoelectric effect is the main factor in attenuation, and with high energy, Compton scattering is the main factor. For this reason, when the lower energy of CT is used for attenuation correction, highatomic-number metals result in attenuation estimates that are more than the actual SPECT attenuation. Therefore, an artificial femoral head and hip joint cause overestimation when CTAC is used.

In this investigation, overestimation occurred when the default attenuation correction formula was used, although the result was better than when no attenuation correction was performed. When the count of locations having metal was compared with the count of locations having no metal, overestimation was in the $8 \%-20 \%$ range, and overestimation in the presence of a $22-\mathrm{cm}$ artificial hip joint was at the maximum, with the paired $t$ test showing a significant difference of $1 \%$. To control this overestimation, thresholds were set for the attenuation coefficient. A $\mu$-map on which 2 thresholds were used assumed the low threshold value to be a bone attenuation coefficient and the high threshold value to be a metal attenuation coefficient. When functioning for making a $\mu$-map, CT values become lower than usual even if in the same tissue. On the original $\mu$-map, 0.20 is a CT value of about 600 and 0.30 is a CT value of about 1,500 . There is usually no tissue other than bone that shows a CT value of 600 in tissue in the living body when the tube voltage is $130 \mathrm{kV}$. The CT value of almost all bones is less than 1,300. Even a threshold of 0.30 creates no problem. If 2 thresholds are set, the attenuation correction formula turns into a hybrid formula incorporating both the bilinear method and the segmentation method. Use of thresholds of 0.20 and 0.30 in the $\mu$-map controlled the overestimation by metal better than did other thresholds. There was less than a 5\% error rate, even if every fitted part was metal. In this experiment, overestimation was controlled regardless of the size or form of the metal. Therefore, a $\mu$-map with 2 thresholds is suitable for suppressing the overestimation.

Since the aim of this investigation was to control overestimations caused by metal, other issues, such as the star artifacts that can occur with metal, were not considered.

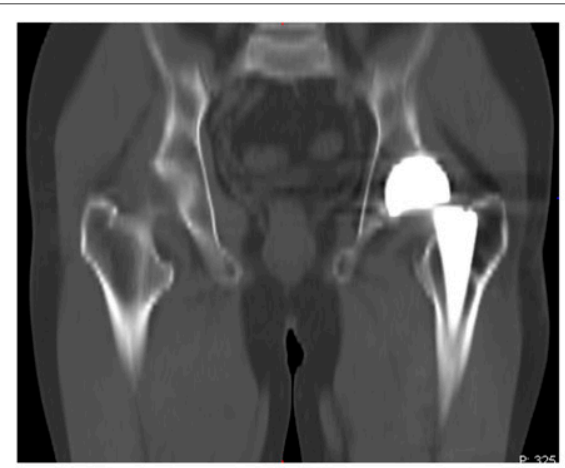

CT

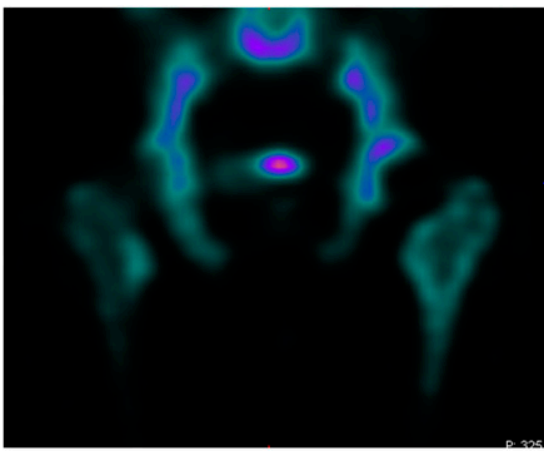

Default

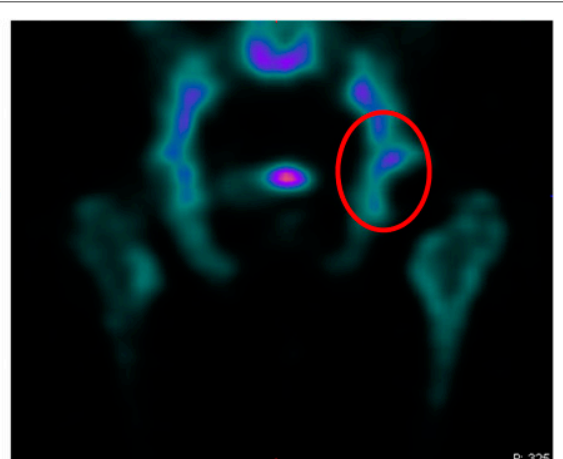

$0.2-0.3$

FIGURE 5. Comparison between image with no threshold and image with 2 thresholds. In circled area, count and appearance changed with thresholds. 
We tested our method on the data of an actual (prior) patient who had an artificial hip joint. Figure 5 compares use of no threshold and use of 2 thresholds in this patient. The count of the stem portion is clearly different, and the images do not differ except when the metal is present. Thus, the potential usefulness of the method in actual patients is seen, although an observer experiment is needed to confirm this usefulness. If application of the method in an actual patient is possible, CTAC in a patient with a metallic implant, such as an artificial femoral head, will be more accurate (9). Moreover, in the presence of metals such as a dental device or a pacemaker, a different threshold may need to be set. If each required threshold value can be determined, the overestimation by metal will vanish. Such determinations will be especially useful if they can control for overestimation due to the lead of the pacemaker in a cardiac examination. Achieving this goal will cancel at least one fault of CTAC.

\section{CONCLUSION}

By using a correction formula that includes 2 threshold values, we succeeded in decreasing from $20 \%$ to $4 \%$ the overestimation of CTAC caused by the presence of metals. The SPECT/CT data produced by this method will have greater accuracy in many patients with metallic implants.

\section{DISCLOSURE}

No potential conflict of interest relevant to this article was reported.

\section{ACKNOWLEDGMENT}

We thank Ikuhiro Shinoda (Siemens Japan K.K.) for assistance with this investigation.

\section{REFERENCES}

1. Rybak LD, Rosenthal DI. Radiological imaging for the diagnosis of bone metastases. Q J Nucl Med. 2001;45:53-64.

2. Blankespoor SC, Wu X, Kalki JK, et al. Attenuation correction of SPECT using $\mathrm{X}$-ray CT on an emission transmission CT system: myocardial perfusion assessment. IEEE Trans Nucl Sci. 1996;43:2263-2274.

3. Patton JA, Turkington TG. SPECT/CT physical principles and attenuation correction. J Nucl Med Technol. 2008;36:1-10.

4. Linke R, Kuwert T, Uder M, et al. Skeletal SPECT/CT of the peripheral extremities. AJR. 2010;194:329-335.

5. Utsunomiya D, Shiraishi S, Imuta M, et al. Added value of SPECT/CT fusion in assessing suspected bone metastasis: comparison with scintigraphy alone and nonfused scintigraphy and CT. Radiology. 2006;38:264-271.

6. Horger M, Eschmann SM, Pfannenberg C. Added value of SPECT/CT in patients suspected of having bone infection: preliminary results. Arch Orthop Trauma Surg. 2007;127:211-221.

7. Lacroix KJ, Tsui BMW, Hasegawa BH, et al. Investigation of use of x-ray CT images for attenuation compensation in SPECT. IEEE Trans Nucl Sci. 1994;41:2793-2799.

8. Amarasekera HW, Costa ML, Parsons N, et al. SPECT/CT bone imaging after hip resurfacing arthroplasty: is it feasible to use CT attenuation correction in the presence of metal implants? Nucl Med Commun. 2011;32:289-297.

9. Chew GC, Lewis P, Middleton F, et al. Radionuclide arthrogram with SPECT/CT for the evaluation of mechanical loosening of hip and knee prostheses. Ann Nucl Med. 2010;24:735-743. 